\title{
Fatal attraction: chemokines and type 1 diabetes
}

\author{
Mark A. Atkinson ${ }^{1}$ and S. Brian Wilson ${ }^{2}$ \\ ${ }^{1}$ Department of Pathology, University of Florida, Gainesville, Florida, USA \\ ${ }^{2}$ Dana-Farber Cancer Institute, Boston, Massachusetts, USA \\ J. Clin. Invest. 110:1611-1613 (2002). doi:10.1172/JCI200217311.
}

Research discoveries within the past few years have dramatically reshaped our collective thought on the pathogenesis and natural history of type 1 diabetes (1). Many concepts once held as dogmas (e.g., B lymphocytes play no pathogenic role in type 1 diabetes; autoantibodies only serve as markers of the disease process; and Th1 cytokines are bad, in terms of the disease, whereas Th2 cytokines are good) have undergone major modification and, in some cases, have been completely reversed. It now appears that $B$ lymphocytes play vital roles in presenting autoantigens necessary for disease development; that eliminating the maternal passage of antibodies can influence the subsequent rate of diabetes in offspring; and that the Th1/Th2 model has been saddled with so many "footnotes" regarding exceptions that it is now clear that initial hopes for a simple model to explain the immunopathogenesis of type 1 diabetes were, unfortunately, unrealistic.

In addition to dogmas falling, many forgotten or overlooked aspects of the

\footnotetext{
Address correspondence to: Mark A. Atkinson, Department of Pathology, Immunology and Laboratory Medicine, College of Medicine, University of Florida, ARB-R3-128, 1600 SW Archer Road, Gainesville, Florida 32610-0275, USA. Phone: (352) 392-0048; Fax: (352) 392-8464; E-mail: atkinson@ufl.edu.

Conflict of interest: The authors have declared that no conflict of interest exists. Nonstandard abbreviations used:

CC chemokine receptor (CCR); CXC chemokine receptor (CXCR); nonobese diabetic (NOD); monocyte chemoattractant protein (MCP); macrophage inflammatory protein (MIP); regulated on activation, normal $T$ cell expressed and secreted (RANTES); IFN-inducible protein-10 (IP-10); thymus- and activation-regulated chemokine (TARC); macrophage-derived chemokine (MDC); glutamate decarboxylase (GAD).
}

immune system as they apply to type 1 diabetes have found new life. Among such old (and even a few new) notions generating increased interest are antigen-presenting cells (e.g., dendritic cells, B lymphocytes), nontraditional $\mathrm{T}$ cells (e.g., NKT cells, NK cells), non-class I and -class II MHC molecules (e.g., CD1, MHC class I chainrelated), cell receptors or intracellular pathways (e.g., toll, suppressor of cytokine signaling), and chemokines. A report by Kim and colleagues in this issue of the JCI investigates the role of chemokines and their receptors in $\mathrm{T}$ cell migration in a mouse model for human insulin-dependent diabetes (2).

\section{Chemokines: calling out the troops}

Chemokines represent a class of cytokines that have chemoattractant properties (3-5). Simply put, cells with the appropriate chemokine receptor (e.g., eosinophils, fibroblasts, leukocytes, monocytes, neutrophils, NK cells, or other effector cells) will migrate to the source of chemokine production and release. Chemokines are produced by a wide variety of cell types in response to infection (e.g., bacterial or viral products) or agents that cause physical damage to a tissue. They are not the sole mediator of cell recruitment, a process that also requires cytokines to induce the expression of endothelial adhesion molecules and vasoactive mediators that promote leukocyte interactions with vessel endothelium. In addition to migration, chemokines display activities influencing angiogenesis, lymphocyte development, and direct defense against infection.

With more than 50 chemokines identified thus far, their nomenclature has, unfortunately, been problematic $(3,6,7)$. Currently, they are classified into groups based on the position of cysteine motifs near the $\mathrm{N}$-terminal portion of the molecule. The two largest families are termed $\mathrm{CC}$ and CXC. CC chemokines have two adjacent cysteines near their amino-terminus, whereas in CXC chemokines, the two cysteines are separated by another amino acid. CC chemokines bind to CC chemokine receptors (CCRs), whereas CXC chemokines bind to CXC chemokine receptors (CXCRs).

\section{Chemokines and type 1 diabetes}

In the type 1 diabetes literature, most reports involving chemokines derive from studies of the nonobese diabetic (NOD) mouse model (8). The earliest association of chemokines with NOD mice was genetic, indicating a central region of mouse chromosome 11 associated with diabetes included what was then called the $\beta$-chemokine gene family (9). Later studies demonstrated that a T cell-proliferative unresponsiveness on chromosome 11 in NOD mice was linked to a disease-associated region known as Idd4, and that within a 5.2centimorgan interval coupled with this phenotype were the CC chemokine genes (10). In terms of chemokine-based immunoregulation, the subpopulation of $\mathrm{CD} 4^{+} \mathrm{CD} 25^{+}$splenocytes capable of delaying the adoptive transfer of diabetes reportedly expresses high levels of the chemokine receptor CCR7 (11). However, the largest number of investigations involving chemokines and NOD mice have examined the site of autoimmune attack - the pancreatic islet. These studies have purported an association between, on one hand, the formation of insulitis and/or progression to disease and, on the other hand, islet cell expression of monocyte chemoattractant protein-1 (MCP-1), macrophage inflammatory protein-1 $\alpha$ (MIP-1 $\alpha$ ), 


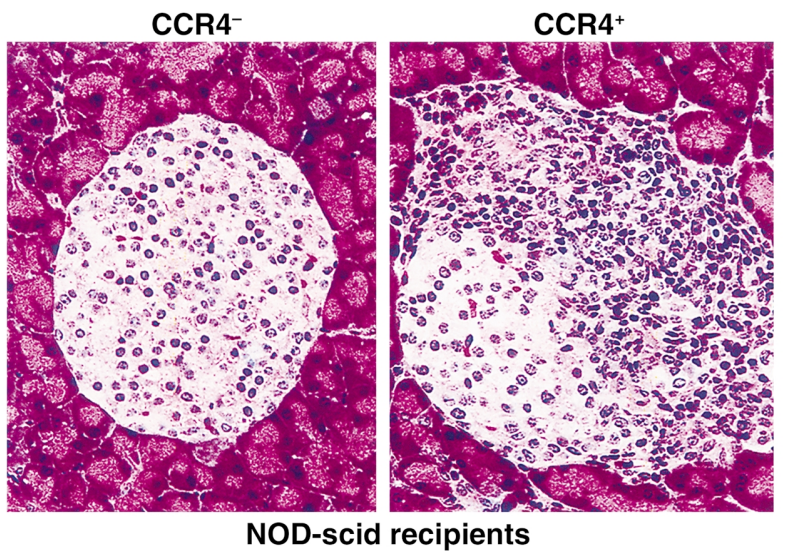

Figure 1

The influence of CCR4 in islet inflammation. Adoptive transfer studies performed by Kim et al. (2) suggest that CCR4-expressing T cells obtained from 16- to 18-week-old diabetic NOD mice confer a higher capacity for imparting pancreatic inflammation (i.e., insulitis) in NOD-scid recipients than do CCR4-negative cells. Indeed, the figure clearly demonstrates a marked degree of infiltration, and disruption of islet architecture was associated with transfer of CCR4-positive cells. It is key to note that this figure should be considered as representative since the quantitative data for insulitis reported in the study were not as striking as those qualitatively demonstrated in this figure in terms of the differences in inflammatory capacity afforded by the two populations of cells. However, this work does support a role of CCR4 in the homing of inflammatory cells to the pancreatic islet. This study provides a conceptual framework for the design of therapeutics aimed at interrupting the formation of insulitis and the eventual development of type 1 diabetes.

MIP-1 $\beta$, CCR5, RANTES (regulated on activation, normal $\mathrm{T}$ cell expressed and secreted), MCP-3, MCP-5, and IFNinducible protein-10 (IP-10) (12-14).

Some studies involving chemokines and humans with type 1 diabetes have been reported, but they are limited in that they focus on activities in peripheral blood. Two recent studies reported elevated serum levels of IP-10, a promoter of migration of activated Th1 cells, in individuals with type 1 diabetes or nondiabetic individuals at increased risk for the disease as defined by the presence of autoantibodies $(15,16)$. Persons with type 1 diabetes also reportedly have increased peripheral blood mononuclear cell expression of chemokine receptors CXCR4 (naive $\mathrm{T}$ cells), CCR5 and CXCR3 (Th1-associated), CCR3 and CCR4 (Th2-associated), as well as serum chemokine levels of MCP-1, MIP-1 $\alpha$, MIP-1 $\beta$, and RANTES (17). These and other studies also suggested reduced expression of the Th1-associated chemokine receptors CCR5 and CXCR3 in type 1 diabetes patients (17). Finally, a genetic association involving a single-base polymorphism in CCR2, a chemokine receptor involved in the trafficking of leukocytes in both basal and inflammatory states, has been described for persons with type 1 diabetes (18).

\section{Th1 ... Th2 ... Th 3 ... and now CCR4 in type 1 diabetes}

Assertions of abnormal cytokine production with type 1 diabetes abound (19) and have often been described as supporting or refuting a Th1/Th2 model for type 1 diabetes. The study by Kim et al. (2), however, goes far beyond previous studies by linking CCR4-bearing $\mathrm{T}$ cells with what is presumably a key facet of the autoimmune destruction of $\beta$ cells: the recruitment of antigen-specific memory $\mathrm{T}$ cell effectors with the tissue-specific accumulation of antigen-presenting cells. Specifically, the investigators demonstrate that neutralization of macrophage-derived chemokines (MDCs) through antibody administration in NOD mice was capable of reducing the degree of insulitis (including a reduction in CCR4-positive $T$ cells) as well as the rate of diabetes. Conversely, studies of MDC transgenic animals suggested that disease acceleration occurred via recruitment of CCR4-positive T cells (2) (Figure 1). Taken collectively, these authors portend that CCR4-positive T cells represent a key factor facilitating the migration of pathogenic $T$ cell populations (i.e., antigen-specific memory $\mathrm{T}$ cell effectors) to the islet lesion.

What cautions should one apply to this study (2)? First, the efficacy of dis- rupting the formation of type 1 diabetes by blocking MDC was incomplete with respect to disease prevention. Potentially, this could be the result of other CCR4 ligands such as thymus- and activation-regulated chemokine (TARC), the ligand for CCR4, subserving the function of MDC. Moreover, the selective expression of TARC in islets and of MDC in the pancreatic lymph node suggests that further investigation of the temporal-spatial regulation of chemokine production might be important for a more complete understanding of the pathogenic mechanisms involved. Caution should also be exercised in evaluating the studies of islet antigen reactivity - in particular, responses to glutamate decarboxylase (GAD) and the BDC 2.5 antigen. The question of whether GAD is even present in murine $\beta$ cells has been the subject of intense debate, and the biochemical nature of the BDC 2.5 antigen remains loosely defined. Finally, in terms of the potential therapeutic value of these studies, one could argue that we have been down this road before (a decade ago?) with a series of investigations suggesting that manipulation of the expression of integrins and adhesions could influence disease progression. The inability of agents to specifically interrupt such processes at a local level (i.e., pancreatic islets) has thus far limited the therapeutic potential of these findings, and such may also be the case for the observations surrounding chemokines and their receptors.

\section{What does it all mean?}

Despite the aforementioned caution regarding therapeutic potential, the findings are timely in that the type 1 diabetes field is actively seeking novel avenues for disease prevention. Type 1 diabetes among autoimmune disorders in that it can be identified months to years before the onset of clinical symptoms by a combination of immunologic and genetic markers (1). A key missing ingredient in attempts to prevent the disease has been a safe yet effective therapy capable of interrupting the autoimmune process in persons at high risk for developing the disease. Theoretically, the migration of peripheral $T$ cells to the pancreatic islets (a process that we presume occurs over a period of months to years before a significant mass of $\beta$ cells is destroyed) could pos- 
sibly be interrupted by targeted disruption of CCR4-bearing T cells.

Besides prevention, another direction for further study is the mechanisms of disease pathogenesis. An interesting comparison between the study by Kim et al. (2) and that of Lohmann et al. (17) is that in the latter study, CCR4 cells were reduced in both newly diagnosed and long-standing type 1 diabetes patients, an association that also correlated to reduced phytohemagglutinin-stimulated IL-4 production. Furthermore, MIP$1 \alpha$ and MIP- $1 \beta$ levels were considerably elevated in a subgroup of patients with newly diagnosed type 1 diabetes (18). In light of these results, one could speculate that Th1-associated peripheral $\mathrm{T}$ cells are reduced in a narrow time window at the time of diabetes diagnosis, possibly due to their extravasation in the inflamed pancreas. Thus, further studies involving longitudinal examination of chemokines and chemokine receptors in the period preceding the natural history of type 1 diabetes appear warranted to evaluate their role in the pathogenesis of the disease and, in addition, to provide evidence for diagnostic utility.
1. Atkinson, M., and Eisenbarth, G. 2001. Type 1diabetes: new perspectives on disease pathogenesis and treatment. Lancet. 358:221-229.

2. Kim, S.H., Cleary, M.M., Fox, H.S., Chantry, D., and Sarvetnick, N. 2002. CCR4-bearing T cells participate in autoimmune diabetes. J. Clin. Invest 110:1675-1686. doi:10.1172/JCI200215715.

3. Baggiolini, M. 2000. Reflections on chemokines. Immunol. Rev. 177:1-5.

4. Proudfoot, A.E.I. 2002. Chemokine receptors: multifaceted therapeutic targets. Nat. Rev. Immunol. 2:106-115.

5. Mellado, M., Rodriguez-Frade, J.M., Manes, S., and Martinez, C. 2001. Chemokine signaling and functional responses: the role of receptor dimerization and TK pathway activation. Annu. Rev. Immunol. 19:397-421.

6. Rossi, D., and Zlotnik, A. 2000. The biology of chemokines and their receptors. Annu. Rev. Immunol. 18:216-242.

7. Murphy, P.M., et al. 2000. International union of pharmacology. XXII. Nomenclature for chemokine receptors. Pharmacol. Rev. 52:145-176.

8. Atkinson, M.A., and Leiter, E.H. 1999. The NOD mouse model of type 1 diabetes mellitus: as good as it gets? Nat. Med. 5:601-604.

9. Gill, B.M., Jaramillo, A., Ma, L., Laupland, K.B., and Delovitch, T.L. 1995. Genetic linkage of thymic T-cell proliferative unresponsiveness to mouse chromosome 11 in NOD mice. A possible role for chemokine genes. Diabetes. 44:614-619.

10. Grattan, M., Mi, Q.S., Meagher, C., and Delovitch, T.L. 2002. Congenic mapping of the diabetogenic locus Idd4 to a 5.2-cM region of chromosome 11 in NOD mice: identification of two potential candidate subloci. Diabetes. 51:215-223.

11. Szanya, V., Ermann, J., Taylor, C., Holness, C., and
Fathman, C.G. 2002. The subpopulation of CD4(+)CD25(+) splenocytes that delays adoptive transfer of diabetes expresses L-selectin and high levels of CCR7. J. Immunol. 169:2461-2465.

12. Bradley, L.M., et al. 1999. Islet-specific Th1, but not Th2, cells secrete multiple chemokines and promote rapid induction of autoimmune diabetes. J. Immunol. 162:2511-2520.

13. Cameron, M.J., et al. 2000. Differential expression of CC chemokines and the CCR5 receptor in the pancreas is associated with progression to type I diabetes. J. Immunol. 165:1102-1110.

14. Chen, M.C., Proost, P., Gysemans, C., Mathieu, C., and Eizirik, D.L. 2001. Monocyte chemoattractant protein-1 is expressed in pancreatic islets from prediabetic NOD mice and in interleukin-1 beta-exposed human and rat islet cells. Diabetologia. 44:325-332.

15. Shimada, A., et al. 2001. Elevated serum IP-10 levels observed in type 1 diabetes. Diabetes Care. 24:510-515.

16. Nicoletti, F., et al. 2002. Serum concentrations of the interferon-gamma-inducible chemokine IP-10/CXCL10 are augmented in both newly diagnosed Type I diabetes mellitus patients and subjects at risk of developing the disease. Diabetologia. 45:1107-1110.

17. Lohmann, T., et al. 2002. Reduced expression of Th1-associated chemokine receptors on peripheral blood lymphocytes at diagnosis of type 1diabetes. Diabetes. 51:2474-2480.

18. Szalai, C., et al. 1999. Chemokine receptor CCR2 and CCR5 polymorphisms in children with insulindependent diabetes mellitus. Pediatr. Res. 46:82-84

19. Rabinovitch, A, and Suarez-Pinzon, W.L. 1998. Cytokines and their roles in pancreatic islet betacell destruction and insulin-dependent diabetes mellitus. Biochem. Pharmacol. 55:1139-1149. 